diathesis, and shock. The mode of transmission and clinical course would vary depending on the specific pathogen. Diagnosis may be delayed given clinicians' unfamiliarity with these diseases, heterogeneous clinical presentation within an infected cohort, and lack of widely available diagnostic tests. The initiation of ribavirin therapy in the early phases of illness may be useful in the treatment of some of these viruses, although extensive experience is lacking. There are no licensed vaccines to treat the diseases caused by HFVs.

FROM: Borio L, Inglesby BL, Peters CJ, et al. Hemorrhagic fever viruses as biological weapons: medical and public health management. JAMA 2002;287:23912405.

\section{OSHA Prohibits Needle Removal and Phlebotomy Tube Holder Reuse}

On June 12, 2002, the Occupational Safety and Health Administration (OSHA) issued a long awaited letter of interpretation to clarify its position on the prohibition of removing contaminated needles from phlebotomy blood tube holders and reusing the tube holders, citing a risk to workers from this practice. The letter of interpretation notes that all tube holders with needles attached must be immediately discarded into a sharps disposal container after the safety feature has been activated. OSHA has explained that this practice helps to prevent potential worker exposure to the contaminated hollow bore needle at both the front and the back ends.

For those facilities that already use single-use blood tube holders, this new interpretation will have little impact. However, facilities that will be affected and that will need to change their practice include those that use phlebotomy safety devices in which the safety mechanism is built into or is part of the needle and that remove the needle after activation of the safety feature and reuse the holder. Many hospitals that are removing needles and reusing tube holders have expressed concern that compliance will increase costs. Others have expressed concern over increased amounts of medical waste and related increased disposal costs. Although this is a new interpretation for OSHA at the federal level, some state OSHA plans (eg, California) have been prohibiting needle removal and phlebotomy holder reuse for some time.

FROM: U.S. Department of Labor/Office of Public
Affairs. OSHA clarifies position on the removal of contaminated needles [press release]. June 12, 2002. Available at www.osha.gov.

\section{Serratia marcescens Bacteremia Traced to an Infused Narcotic}

The Centers for Disease Control and Prevention's Division of Healthcare Quality Promotion, National Center for Infectious Diseases, conducted an investigation of an outbreak of Serratia marcescens bacteremia among several patients in a surgical intensive care unit between June 30, 1998, and March 21, 1999. The outbreak was traced to extrinsic contamination of the parenteral narcotic fentanyl by a healthcare worker.

To identify risk factors, patients with $S$. marcescens bacteremia were compared with randomly selected controls. Isolates from patients and from medications were evaluated by pulsed-field gel electrophoresis. The hair of one employee was tested for fentanyl.

Twenty-six patients with $S$. marcescens bacteremia were identified; 8 (31\%) had polymicrobial bacteremia, and 7 of these had Enterobacter cloacae and S. marcescens in the same culture. According to univariate analysis, patients with $S$. marcescens bacteremia stayed in the surgical intensive care unit longer than did controls (13.5 vs 4.0 days, $P<$ .001 ), were more likely to have received fentanyl in the surgical intensive care unit (odds ratio, $31 ; P<.001$ ), and were more likely to have been exposed to two particular respiratory therapists (odds ratios, 13.1 and $5.1 ; P<.001$ for both comparisons). In a multivariate analysis, receipt of fentanyl and exposure to the two respiratory therapists remained significant. One respiratory therapist had been reported for tampering with fentanyl; his hair sample tested positive for fentanyl. Cultures of fentanyl infusions from two case patients yielded $S$. marcescens and $E$. cloacae. The isolates from the case patients and from the fentanyl infusions had similar patterns on pulsed-field gel electrophoresis. After removal of the implicated respiratory therapist, no further cases occurred.

This investigation underscores the risk of complications in patients that is associated with illicit narcotic use by healthcare workers.

FROM: Ostrowsky BE, Whitener C, Bredenberg HK, et al. Serratia marcescens bacteremia traced to an infused narcotic. $N$ Engl J Med 2002;346:1529-1537. 